\title{
On Family Tree of Transition Elements
}

\author{
Zhifu Wu $\mathrm{W}^{1,2, \mathrm{a}^{*}}$, Liyun $\mathrm{Hu}^{3, \mathrm{~b}}$ and Qinglin $\mathrm{Han}^{1, \mathrm{c}}$
}

1 School of Materials Science and Engineering, Baise University, Baise 533000, China

2 Guangxi Colleges and Universities Key Subject of Material Physics and Chemistry, Baise University, Baise, 533000, China.

3 Library of Baise University, Baise, 533000, China.

a1781177844@qq.com; b719370462@qq.com; '417507785@qq.com

*Corresponding author: 1781177844@qq.com 86-776-2848131

\section{Keywords: Transition elements; Definition; Classification; Aesthetics}

\begin{abstract}
With the extensive applying of long periodic table, the definition of historical transition elements has become styles nowadays from narrow to wide. How ever there are three methods to define transition elements and several sorts of classifying items .It is very important to unify its definition and classification like mapping out International System of Unit (SI)
\end{abstract}

\section{Introduction}

The chemical periodic table should be considered as the most important concept both in theory and in practice. And it is what the students need in their daily study, and also provides new ways of doing research for professional persons. What's more, it has concisely arranged the whole process of chemical transformation. From the chemical periodic table, the fact has been obviously proved that chemical elements are not disorderly and unsystematic matters, yet they have complex relations between each other. And each cluster made up of certain elements gather together, showing various trend. Thus it is quite important to know about the periodic table for anyone who eager to recognize that how the world is comprised of the element which is a basic structural unit in chemistry. And anyone that tries to get familiar with our world with an insight of scientists should at first get a complete picture of the periodic table, for it has become an integrate part of scientific culture. ${ }^{[1]}$

Within rich and varied knowledge in chemistry, The chemical periodic table which consisting of a great many elements that like numbers of the entire family, is the basic essential tool to professional persons working on chemistry, the transition elements is the biggest branch of that family. However, since the announcement of the Mendleeve's periodic table and other forms of periodic table, there have been different definitions of cropped up transition elements, leading to the confused problem with coexistence of various definitions. For which the research work and study are frustrated or with many years' teaching experiences, the author puts forward the definition organic combination o both the complete picture of the periodic table and the macro properties of elements, and takes the transition elements as the most appropriate definition. In the end, it is also suggested that the transition element should be defined in the 18-duster periodic table introduced by IUPAC, and systematizing and simplifying that definition.

\section{On the Definition of Transition Elements}

\section{Coexistence of Varieties of Definitions}

Since the Mendeleev's periodic table of chemical elements was announced in 1869, scores of kinds of periodic tables have been published in succession, among which, the short table and the long table are two most general forms. At early time, when the transition elements were advanced, they only referred to the nine elements both in cluster iron and cluster platinum in the periodic table. With the extensive application of long periodic table, the definition of historical transition elements has become styles nowadays from marrow to wide. The kind of elements, occupying about 80 percent of the total number of elements in the periodic table, including those in transition zone with 
the ranges extended typical mental elements to typical nonmetal elements. Still there are different definitions of those elements in present books. The following are three common ways of defining transition elements. First, some part the orbit $d$ is full. Those transition elements have the atom whose characteristic electron shell structure is $(n+1) d^{1-9} n s^{1-2}$ For instance, in book 2 of Inorganic Chemistry taken for higher school education and edited by Beijing Normal University . The transition element is defined as elements in zone block $\mathrm{d}$ from cluster IIIB to cluster VIIIB, in which the elements of Cluster Lanthanum except La and of cluster actinium except Ac are not included. ${ }^{[3]}$ Second, the atoms or part of the orbit $\mathrm{d}$ of its ion is partly filled. Its characteristic electron structure is $(n-1) d^{1-9} \mathrm{~ns}^{1-2}$, form transition elements include cluster cuprum, but not cluster Zinc, for the structure of the main oxidation state of cuprum namely $\mathrm{Cu}^{2+}$, is $3 \mathrm{~d}^{9}$, while that of Zinc, namely $\mathrm{Zn}^{2+}$, is $3 \mathrm{~d}^{10}$ and fully filled. This method of defining is adopted in the book. Inorganic Chemistry written by R.B. Heslop and K.Jones. ${ }^{[4]}$ Third, all the elements in sub clusters, and they are such transition elements with atoms whose characteristic electron structure is $(n-1) d^{1-10} \mathrm{~ns}^{1-2}$, including both cluster $\mathrm{Cu}$ and Cluster $\mathrm{Zn}$. This definition has been adopted in the following two books, Common Inorganic Chemistry ${ }^{[5]}$ compiled by Xuanshen Yan and Changfu Wang and. Advanced Inorganic Chemistry ${ }^{[6]}$ Co-written by F.A.cotton from America and G.Wilkson from Britain .To sum up ,the main controversy focuses on the elements in cluster $\mathrm{Cu}$ and cluster $\mathrm{Zn}$ of zone ds both of them, partly belong to transition elements or completely belong to .According to the teaching experiences over many years, the writer assents to the third method of definition.

\section{The Cause for Unity}

Firstly, the previous definition of transition elements hasn't cornered with the microstructure of chemical elements' while the difference of microstructure between elements can be denoted by dividing them into different blocks, such as block s, $\mathrm{p}, \mathrm{d}$, ds and zone $\mathrm{f}$, among which elements in zone $\mathrm{d}$, $\mathrm{ds}$ and $\mathrm{f}$ are all transition elements, And the properties of elements in zone $\mathrm{d}$ and zone $\mathrm{ds}$ certainly differ from each other because of their microstructures. But most physical properties of the elements in zone ds are similar to those in zone d. In Inorganic Chemistry, the general properties of transition elements are usually described as below, they are all metals, with high melting point, high boiling point, of strong hardness and fine extensibility, and possess food electrical conductivity and food properties of heat conduction, and they can form alloys between each other, in which $\mathrm{Hg}$, forms alloy nearly with any other metal except with elements in cluster iron, and they are also apt to form coordination compounds. Although the element of cluster zinc has weak hardness, low melting point and low boiling point, it is a special example ,just as rules of Hund. Anything possesses its own specific property and common property. So this way of defining transition elements helps to grasp the common character and connection of elements properties entirely. Seeing from the above properties of transition elements, there are not so many specific examples. Yet they cover a wide range. On chemical properties, the mental reactivity of the elements in zone ds weakens in order of precedence from up to down by analyzing acidity and alkalinity of the oxyhydrogen compound and its law of transformation, it's the same with while it is opposite to those in zone s. It follows that elements in zone ds, which includes cluster Cuprum and cluster zinc, are more similar to any other transition elements than to those in zone $\mathrm{s}$ in chemical and physical properties. Thus are classify elements in zone ds into the family of transition elements right according to their different specific properties.

Secondly, the third definition helps to grasp elements properties macroscopically. At present, there has been more specific classification of modern science. If some subject was compared to an elephant, the academic research would be deep into its legs, toes and even it's teeth. Once buried in blindly, one may be overlooks the mastery of natural Characteristics of matters from the whole, or forgets that what he is researching into is still an elephant, and such a foolish mistake as the blind trying to size up an elephant. More over, we usually do research in one matter or one kind of element beginning with its measurable properties, then according to which we infer its structure. Though the structure determines its property, yet the research into properties can't be conducted by 
the unknown structure. As long as the structure is fixed on through experiments, it can be applied to the research into properties. There for, to define transition element should depend mainly on their general similarities.

Thirdly, with this definition, the periodic table looks entirely pleasing to the eye. Until now, there has been 112 Rinds of elements in the periodic table. Chemists designed them in various patterns, long, short, cylinder-shaped, tower-shaped, fan-shaped and so on. There are 20 illustrations and 80 sorts of tables ${ }^{[7]}$ in the book. The periodic system of Elements written by Shi-feng Li and Pin-jing Li. Taking the most common long periodic table for example, it has been delicately classified looking clear, beautiful and well arranged. Nobel prize winner: Dr Zhen-ning Yang once said, The aesthetics of science can be described with words like harmony. Elegance, Correspondence, plainness and orderliness If we divide out $\mathrm{Pd}\left(4 \mathrm{~S}^{10}\right)$ or $\mathrm{Hg}$ cliquid state) following the former two classifying methods, the periodic table will seen fragmented . In addition, the divisional blocks of elements in the long periodic table are more often made up of rectangle. When the long side of the rectangle stays in horizontal position, it looks table, while the long side is in vertical position, it will appear forceful, sublime and solemn enough. If we divide two dements out from the tower-shaped periodic table, it seems two stones were moved away from the pyramid; If in fan-shaped periodic table, the lack of two element can make continuous curved line and zones break apart. Prompted by the idea of pursuing the perfect, people nave discovered the elements on the hypothesis of Mendeleev's such as Borom-like (Scandium), Aluminium-Like (Gallium) Silicon-like (Germanium), which has filled the vacancies in the table. Gradually people have discovered new elements and have increased the new numbers of the family of element.

In the same way, the elements in block can also be considered as a small periodic table, Element lanthanum should be involved In Group Lanthanide, and so should element Actinium.

\section{Classifications}

In the writer's opinion, it is better to classify the transition element's into two kinds, main transition elements and internal transition elements. Some one classifies them into series one to series three, but actually it is unnecessary. Moreover the series confuse with the number of elements' period. Another classification into light the transition elements and heavy transition elements seems undoubtedly to paint the lily, because it is an obvious characteristic of the periodic table that under elements are heavier than those above, and also the foundation of the invention of periodic table. Angles said, A subject of science becomes mature only when it has completely utilized math's. So when we refer to in the fourth transition elements periodic, Anyone who learns chemistry can easily understand which elements they are without any belligerence of meanings while it will not be definite if we use the first series or the light transition elements. The classification like the series second or third and light. Heavy transition elements only make the table more complicated. Further more, to emphasize the division of lightness and heaviness is scornful of the periodic table.

\section{Conclusion}

The 11th meeting of International Measuring Unit suggested and approved the SI, and that has promoted the economic and cultural intercourse among people all over the world and has solved the historical problem of confused usage of lots of different units.

Also, the SI has such special features as scientific, accurate, concise and practical, and brings great convenience to people, fully embodying the level of modern science and technology. In 1986 the IUPAC took the 18-group periodic table introduced by the book "Nomenclature of Inorganic Chemistry" as the formal table. And this form of table avoided the confused usage of different ordinal signs among many countries and made full use of Arabic numerals, world's mathematical language. And it also abandoned the combination form of Roman numeral and English alphabet. Different Sighs like a star and a triangle have also been used to mark elements in the group La and the group Ac, Based on the above method, it becomes much easier to define the transition elements which covers from group 3 to group 12. Of course, this is the most simple and clear definition. 


\section{Acknowledgements}

This work is supported by the Aluminum alloy technology cooperation education platform of Baise University.

\section{References}

[1] S. F Li, P. J. Li. The peoridic System of Elements. Press of Chengdu Science and Technology University , 1984.

[2] Peng L, Xiaoping Z. Social Stratification and Cooperative Behavior in Spatial Prisoners' Dilemma Games [J]. PLOS ONE, 2015, 10(7):e0131005.

[3] W. X. Zhu, L. M. Liu. Intermediate Inorganic Chemistry. Beijing Normal University Press, 1993. 422

[4] Inorganic Chemistry. The Teaching and Research Section in Beijing Normal University, Nanjing Normal University edit, Higher Education Press. Beijing: 1992. 819

[5] R. B. Heslop, K. Jones Intermediate Inorganic Chemistry. A Guide to Advanced Study Elsevier Scientific Publishing Company, 1976.1

[6] X. S. Yan, C. F. Wang. Common Inorganic Chemistry 2nd edition, Peking University Press, 1999. 184

[7] F. A Cotton, G.Wilkinson, Advanced Inorganic Chemistry, John Wiley \& Sons, Inc. 1972

[8] W .C. Fernelius and W . H. Powell, J, Chem. Educ. 59,504. 1982.

[9] Deane W. Curtin, The Aesthetic Dimension of science, Philosphical Library. N. Y. 1998.

[10] IUPAC, Nomenclature of Inorganic Chemistry $2^{\text {nd }}$ Ed, Butterworths, London .1970.

[11]R.L .Rawls. Revsions to periodic table spark controversy, Jan.27, 1986 Chem. \&Eng. News 